\title{
The Role of SPARC Protein Expression in the Progress of Gastric Cancer
}

\author{
Lifeng Wang • Miling Yang • Lihui Shan • Lei Qi • \\ Cuicui Chai • Qiufeng Zhou $\cdot$ Ke Yao $\cdot$ Hongmei Wu \\ Wenguang Sun
}

Received: 17 April 2011 / Accepted: 3 January 2012 / Published online: 13 January 2012

(C) The Author(s) 2012. This article is published with open access at Springerlink.com

\begin{abstract}
We aimed to investigate the expression of SPARC (secreted protein, acidic and rich in cysteine) in gastric cancer and its relationship with tumor angiogenesis and cancer cells proliferation. Protein expression of SPARC, VEGF, CD34 and Ki-67 in 80 cases of gastric cancer and 30 cases of normal gastric tissue was evaluated by immunohistochemistry. CD34 staining was used as an indicator of microvessel density (MVD). Ki-67 labeling Index (LI) indicated cancer cells proliferation. Statistical analysis was used to investigate its relationship with clinical characteristics, tumor angiogenesis and cancer cells proliferation. SPARC expression was mainly in the stromal cells surrounding the gastric cancer cells, and was statistically significant differences between gastric cancer and normal gastric tissue $(P<$ $0.05)$. Both the expression of SPARC and VEGF were related to differentiation degree, clinical stage, Lauren classification and lymph node metastasis $(P<0.05)$. Expression of SPARC was significantly negatively correlated with the expression of VEGF and MVD in gastric cancer tissues. Expression of SPARC was also negatively correlated with Ki-67-LI. Our findings suggest that both the expression of
\end{abstract}

L. Wang $(\bowtie) \cdot$ L. Shan $\cdot$ L. Qi $\cdot$ C. Chai $\cdot$ Q. Zhou $\cdot$ K. Yao

H. Wu

Department of Pathology, The First Clinical College

of Harbin Medical University,

Harbin 150001, China

e-mail: hljwlf@yahoo.cn

M. Yang

Department of Pathology, The People's Hospital of Zhengzhou, He Nan 450003, China

W. Sun

Department of Nutrition, The First Clinical College

of Harbin Medical University,

Harbin 150001, China
SPARC and VEGF are closed to tumor angiogenesis in gastric cancer, SPARC inhibited tumor angiogenesis but VEGF promoted tumor angiogenesis. SPARC also inhibited cells proliferation of gastric cancer.

Keywords Gastric cancer · SPARC · Angiogenesis · Cancer cells proliferation

\section{Introduction}

In recent years, although the worldwide incidence of gastric cancer has decreased, both its incidence and mortality rate are high in China [1]. The high mortality is closely related to tumor invasion and metastasis. Angiogenesis plays an important role in the survival of the malignant cells, in the local expansion and tumor invasion, as well as in the appearance of distant metastasis. Angiogenesis is not an active process by itself, and it is controlled by some angiogenic factors and some inhibitors of angiogenesis [2]. Of all the angiogenic factors, vascular endothelial growth factor (VEGF) is a potent, multifunctional cytokine that exerts several important and possibly independent actions on vascular endothelium. That is its property and capacity to induce angiogenesis, which has excited the greatest interest in VEGF [3]. Some studies demonstrated that SPARC is related to the invasion, metastasis, angiogenesis and growth of malignant tumor. Other studies found that SPARC could bind with VEGF and decrease the capability of VEGF binding with its receptors, and resulted in the inhibintion of endothelial cell proliferation [4-6].

The purpose of this study was to investigate the relationship between the expression of SPARC with angiogenesis and cancer cells proliferation, as well as the relationship with other clinicopathological parameters in gastric cancer. 


\section{Materials and Methods}

Patients and Tissue Speciments

Eighty cases of gastric cancer obtained between January 2008 and April 2009 were identified from our pathology files in Department of Pathology at the Fourth Clinical Hospital of Harbin Medical University, China. The study group comprised 59 male and 21 female patients, range $35-83$, median age 61 . The clinicopathological parameters were obtained from the pathological reports, including tumor differentiation, lymph node metastasis, TNM stage and Lauren classification, and all of these data were reviewed and confirmed by the experienced pathologists in our department. Thirty cases of corresponding nontumorous tissue samples were collected as controls.

Immunohistochemical Staining and Scoring

Sections $(4 \mu \mathrm{m})$ from the paraffin-embedded, formalinfixed gastric cancer tissues were fixed on the charged slides for immunohistochemical analysis using non-biotin detectionsystem (EliVision. Anti-Mouse/Rabbit-HRP, DAKO). Primary mouse monoclonal antibodies to SPARC (clone NCL-O-NECTIN, Novocastra), CD34 (clone QBEnd/10,DAKO Carpinteria, USA), primary rabbit monoclonal antibodies to VEGF (clone SP28, Zhongshan) and primary mouse monoclonal antibodies to Ki-67 (clone SP6, Zhongshan) were used in the study. All slides were deparaffinized with xylene and rehydrated through graded concentrations of ethanol ending with distilled water. Then endogenous peroxidase was blocked by $0.3 \%$ hydrogen peroxide for $10 \mathrm{~min}$. Sections for SPARC,VEGF, CD34 and Ki-67 for immunohistochemical were subjected to microwave antigen retrieval with $0.1 \mathrm{M}$ citrate buffer $(\mathrm{pH} 6.0)$ at $800 \mathrm{~W}$ for $5 \mathrm{~min}$, and slides were cooled for $20 \mathrm{~min}$ at room temperature and washed in PBS $(\mathrm{pH} 7.4)$, then were incubated $1 \mathrm{~h}$ at $37^{\circ} \mathrm{C}$ in humidified chambers, followed by EliVision detection incubated for $20 \mathrm{~min}$ at room temperature. The staining were visualized by incubating with 3,3'-diaminob-enzidine for $3 \sim 5 \mathrm{~min}$, then counterstained with hematoxylin. Sections of known positive specimens were used as positive controls. Sections incubated with PBS instead of primary antibody were used as negative controls.

The intensity of immunostaining for SPARC was reviewed and scored according to the location of cytoplasmic and results are presented by two independent observers without knowledge of the clinicopathological parameters of the patients. The proportion of cells with SPARC expression was rated as follows: 0 point, $\leq 5 \%$ positive stromal fibroblast cells; 1 points, $6 \% \sim 25 \%$ positive cells; 2 points , $26 \% \sim 50 \%$ positive cells; 3 points, $\geq 51 \%$ positive cells. The intensity of staining varied from weak to strong. The intensity was classified as a scale of 0 (no staining), 1 (weak staining, light yellow), 2 (moderate staining, yellowish brown), and 3 (strong staining, brown). Staining index was calculated as the product of staining intensity score and the proportion of positive stromal cells. We obtained the staining index with scores of $0,1,2,3,4,6$,or 9 , a staining index score $\geq 4$ was used to define stromal cells with high SPARC expression, and a staining index score $\leq 3$ was used to indicate low SPARC expression [7].

The staining results of VEGF were evaluated by a score corresponding to the sum between: the percentage of cytoplasmic positive tumor cells [8]: 0 point, $0 \%$ immunopositive cells; 1 point, $\leq 25 \%$ positive cells; 2 points, $26 \sim 50 \%$ positive cells; 3 points, $\geq 51 \%$ positive cells; the staining intensity: 0 point, negative immunoreaction; 1 point, weak intensity; 2 points, moderated intensity; 3 points, strong intensity. The sum of the two parameters varied between 0 and 6: a negative immunoreaction (-) for a score $0 \sim 2$, and a positive immunoreaction ( + ) for a score $3 \sim 6$.

MVD was assessed according to the criteria proposed by Weidners et al. [9], the brownish cytoplasmic staining of vascular endothelial cells was considered positive. The microvessels were counted according to the number of single endothelial cell or endothelial cell cluster showing brownish yellow granules in the cytoplasm. The sections were observed first under the low-power $(\times 40)$, then the most dense area of microvessel sections was selected under the high-power $(\times 400$, the surface area of every vision field being $0.785 \mathrm{~mm}^{2}$ ). The number of microvessels in three vision fields were counted and averaged as MVD of the given specimen.

The positive staining of Ki-67 was nuclear staining. Five areas of highest proliferative activity were selected randomly at low magnification. Then tumor cell counts were performed with these areas at a magnification of $\times 400.100$ tumor cells were counted per field. Ki-67-LI was defined as the percentage of tumor cell nuclei staining [10].

\section{Statistical Analysis}

SPSS 11.5 was used for the statistical analysis. $\chi^{2}$ test was used for testing numeration data and $t$ test was used to test measurement data. Spearman rank correlation coefficient test analysis was performed to examine the correlations between SPARC and VEGF. Mann-Whitney $U$-test was used to assess the association between MVD with the expression of SPARC and VEGF. We also used logistic regression analysis. A value of $P$ less than 0.05 was considered statistical significant. 


\section{Results}

Expression of SPARC, VEGF, CD34 and Ki-67 in Gastric Cancer and Normal Gastric Mucosa Tissue (Table 1)

Expression of SPARC protein was detected by immunohistochemistry staining in 80 cases of gastric cancer tissues and 30 cases of corresponding nontumorous tissues. SPARC was mainly localized in the cytoplasm of stromal cells surrounding the gastric cancer (Fig. 1a). In this study the positive expression of SPARC was 61 cases (76.3\%) in gastric cancer, but in normal gastric mucosa tissue it was only 6 cases $(20 \%)$. SPARC expression was significant difference between the positive rate in stromal cells of gastric cancer and normal gastric mucosa tissue $\left(\chi^{2}=10.8\right.$, $P=0.001$ ).

The positive staining for VEGF was brown granules in the cytoplasm of gastric cancer cells (Fig. 1b) and normal gastric mucosal epithelial cells. The rate of positive VEGF expression was $66.3 \%$ in gastric cancer cells and $30 \%$ in normal mucosal epithelial cells respectively, with a significant difference between them $\left(\chi^{2}=\right.$ $8.45, P=0.004$ ).

MVD any single brown-stained cell or endothelial cell cluster that marks endothelial cell stained with CD34 was counted as a single vessel (Fig. 1c).The mean value of MVD was $43.13 \pm 9.88$ in tumor tissue, and MVD in tumor cells nest was significantly higher than that in the normal mucosal tissue $(t=8.52, P<0.001)$. The positive staining for $\mathrm{Ki}-67$ was buffy granules in the nuclei of gastric cancer cells (Fig. 1d).The mean value of Ki-67-LI was $6.17 \pm 1.22$ in normal mucosal epithelial cells, but it was $22.81 \pm 7.12$ in gastric cancer cells, the difference was significant $(t=12.55$, $P<0.001)$.

Relationship of SPARC, VEGF, MVD and Ki-67-LI with Clinicopathologic Parameters (Table 2)

SPARC expression in stromal cells surrounding the tumor cells nest was statistically significant difference with differentiation degree, clinical stage, Lauren classification and lymph node metastasis $(P<0.05)$, but no significant difference with the patients age, gender, and depth of tumor invasion. But we found SPARC expression was most closed

Table 1 Expression of SPARC, VEGF, CD34 and Ki-67 in gastric cancer and normal gastric mucosa tissue

\begin{tabular}{llllll}
\hline groups & $n$ & SPARC & VEGF & $\operatorname{MVD}(\bar{x} \pm s)$ & $\operatorname{Ki}-67(\bar{x} \pm s)$ \\
\hline normal & 30 & $6(20 \%)$ & $9(30 \%)$ & $23.16 \pm 9.15$ & $6.17 \pm 1.22$ \\
cancer & 80 & $61(76.3 \%)$ & $53(66.3 \%)$ & $43.13 \pm 9.88$ & $22.81 \pm 7.12$ \\
\hline
\end{tabular}

to differentiation degree $(P=0.001)$ by logistic regression analysis.

VEGF expression in the gastric cancer cells was statistically significant difference with differentiation degree, clinical stage, Lauren classification and lymph node metastasis $(P<0.05)$, but no significant difference with the patients age, gender, and depth of tumor invasion. We used logistic regression analysis to assess the relative importance between VEGF and clinicopathologic parameters, and we found Lauren classification was most important.

In gastric cancer, MVD was significantly related to the differentiation degree, clinical stage, depth of tumor invasion, Lauren classification and lymph node metastasis $(P<0.05)$, but MVD was most related to differentiation degree and clinical stage by logistic regression analysis.

The mean value of Ki-67-LI in gastric cancer was associated with depth of tumor invasion, clinical stage and lymph node metastasis $(P<0.05)$, but not with differentiation degree, age, gender and Lauren classification. But we found the clinical stage is the most important by logistic regression analysis.

\section{Correlation Analysis of SPARC Expression and VEGF Expression (Table 3)}

Using Spearman correlation analysis, SPARC expression in stromal cells of gastric cancer was negative significantly related with VEGF expression in cancer cells $(r=-0.344$, $P=0.002)$.

Correlation Analysis of SPARC and VEGF Expression with MVD (Table 4)

In gastric cancer with high SPARC expression, the mean value of MVD was $40.35 \pm 8.35$, but in the gastric cancer with low SPARC expression it was $50.15 \pm 14.33$, with a significant correlation between them $(P=0.001)$. As the SPARC expression became less and less, the mean value of MVD gradually increased.

The mean value of MVD was $49.13 \pm 12.95$ in the positive VEGF expression tumor tissue, and the mean value of MVD was significantly higher than that in the negative VEGF expression tumor tissue $(P<0.001)$.

Correlation Analysis of SPARC Expression and Ki-67-LI (Table 5)

In eighty cases of gastric cancer, the mean value of Ki-67-LI was $24.62 \pm 8.52$ with high SPARC expression, but it was $28.41 \pm 8.29$ with low SPARC expression. It was significantly different between them $(P=0.048)$. As the SPARC 

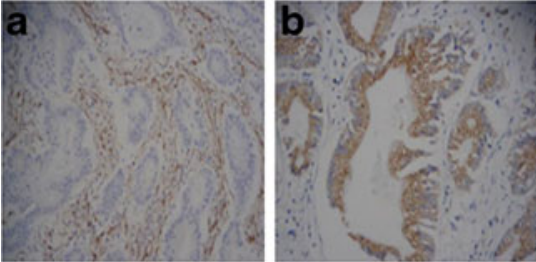

Fig. 1 The expression of SPARC, VEGF, CD34 and Ki-67 in gastric cancer a. High expression of SPARC in stromal cells of intestinal gastric cancer; b. Strong expression of VEGF in the cytoplasm of

expression became less and less, the ability of cancer cells proliferation gradually increased.

\section{Discussion}

Gastric cancer is one of the most common cancer-related cause of death in China. Most gastric cancer is diagnosed at stage III or IV, and the rate of lymph node metastasis or distant metastasis is higher. The invasion and metastasis of tumor is a multifactorial and multistep process. In this process, the formation of blood vessels through endothelial cell proliferation from extant vasculature (angiogenesis) is a prerequisite [11]. Angiogenesis is regulated by multiple proand anti-angiogenic factors produced by various cell types,
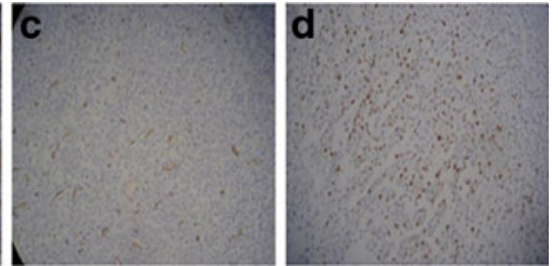

gastric cancer cells; c. Expression of CD34 in endothelial cells of gastric cancer vessels; d. Ki-67 staining was in the nuclei of gastric cancer cells

such as cancer, endothelial and stromal cells. The characteristic of tumor growth is cells proliferation overpass cells death, at the same time, differentiation is inhibited. Cancer cells proliferation are caused by genome inreversible change. In the process of cancer cells proliferation, proand anti-growth gene is essential.

Secreted protein acidic and rich in cysteine (SPARC, also known as osteonectin or BM-40) belongs to the matricellular family of secreted proteins [12]. SPARC is a nonstructural component of extracellular matrices that modulates cell-matrix interactions, particularly during tissue development, remodeling and repair [13]. Recent studies have revealed other biological functions including cell proliferation, migration, deadhesion, antiproliferation, differentiation, and angiogenesis [14]. Some studieds demonstrated that SPARC was related with many

Table 2 Relationship of SPARC, VEGF expression, MVD and Ki-67 with clinicopathologic parameters

\begin{tabular}{|c|c|c|c|c|c|c|c|c|c|c|}
\hline \multirow[b]{2}{*}{ parameters } & \multirow[b]{2}{*}{$n$} & \multicolumn{2}{|c|}{ SPARC } & \multirow[b]{2}{*}{$P$} & \multirow{2}{*}{$\begin{array}{l}\text { VEGF } \\
(+)(-)\end{array}$} & \multirow[b]{2}{*}{$P$} & \multirow{2}{*}{$\operatorname{MVD}(\bar{x} \pm s)$} & & \multirow{2}{*}{$\mathrm{Ki}-67(\bar{x} \pm s)$} & \multirow[b]{2}{*}{$P$} \\
\hline & & high & low & & & & & $P$ & & \\
\hline \multicolumn{11}{|l|}{ age } \\
\hline$<50$ & 12 & 7 & 5 & 0.531 & 102 & 0.175 & $45.33 \pm 15.92$ & 0.980 & $24.76 \pm 8.30$ & 0.446 \\
\hline$\geq 50$ & 68 & 33 & 35 & & 4325 & & $45.23 \pm 12.12$ & & $26.82 \pm 8.64$ & \\
\hline \multicolumn{11}{|l|}{ gender } \\
\hline male & 59 & 28 & 31 & 0.446 & 3722 & 0.262 & $45.76 \pm 12.44$ & 0.547 & $27.49 \pm 8.48$ & 0.086 \\
\hline female & 21 & 12 & 9 & & 165 & & $43.80 \pm 13.42$ & & $23.75 \pm 8.40$ & \\
\hline \multicolumn{11}{|l|}{ Laurentype } \\
\hline intestinal & 38 & 24 & 14 & 0.025 & 1820 & 0.001 & $38.71 \pm 9.21$ & 0.000 & $25.66 \pm 8.90$ & 0.402 \\
\hline diffuse & 42 & 16 & 36 & & 357 & & $51.17 \pm 12.50$ & & $27.28 \pm 8.29$ & \\
\hline \multicolumn{11}{|c|}{ differentiation } \\
\hline high & 41 & 28 & 13 & 0.001 & 2120 & 0.004 & $39.29 \pm 9.06$ & 0.000 & $25.19 \pm 8.01$ & 0.157 \\
\hline low & 39 & 12 & 27 & & 327 & & $51.51 \pm 12.95$ & & $27.91 \pm 9.01$ & \\
\hline \multicolumn{11}{|l|}{ stage } \\
\hline I-II & 41 & 26 & 15 & 0.014 & 2318 & 0.049 & $38.76 \pm 7.05$ & 0.000 & $23.86 \pm 8.52$ & 0.004 \\
\hline III-IV & 39 & 14 & 25 & & 309 & & $52.08 \pm 13.68$ & & $29.30 \pm 7.79$ & \\
\hline \multicolumn{11}{|l|}{ Tumor } \\
\hline $\mathrm{T} 4$ & 50 & 24 & 26 & 0.644 & 3515 & 0.360 & $48.98 \pm 13.40$ & 0.000 & $28.52 \pm 7.77$ & 0.001 \\
\hline T1-T3 & 30 & 16 & 14 & & 1812 & & $39.03 \pm 8.30$ & & $23.16 \pm 8.91$ & \\
\hline \multicolumn{11}{|l|}{$\mathrm{LN}$} \\
\hline N1-N3 & 48 & 18 & 30 & 0.006 & 3612 & 0.043 & $49.94 \pm 13.70$ & 0.000 & $28.49 \pm 8.29$ & 0.010 \\
\hline No & 32 & 22 & 10 & & 1715 & & $38.22 \pm 6.21$ & & $23.54 \pm 8.23$ & \\
\hline
\end{tabular}


Table 3 Correlation analysis of SPARC expression and VEGF expression

\begin{tabular}{|c|c|c|c|c|}
\hline \multirow[t]{2}{*}{ VEGF } & \multicolumn{2}{|c|}{ SPARC } & \multirow[t]{2}{*}{$\mathrm{r}$} & \multirow[t]{2}{*}{$\mathrm{P}$} \\
\hline & high & low & & \\
\hline positive & 20 & 33 & -0.334 & 0.001 \\
\hline negative & 20 & 7 & & \\
\hline
\end{tabular}

tumors, but its role was different depending on the tumor types. Watkins, et al. [15] showed that high levels of SPARC expression in tumor cells negatively correlated with the overall survival of patients in breast cancer. However, the increased SPARC expression in bladder cancer [16] and nonsmall cell lung cancer [17] indicated a higher malignancy and invasion of tumors with poor prognosis. N Said, et al. [18] demonstrated that both tumor and stromal SPARC are limiting for primary prostate tumorigenesis and progression, through effects on the cell cycle.

To explore if SPARC promotes or inhibits the development and progression of tumor, we used immunohistochemical method to detect the expression level of SPARC in human gastric cancer tissues and their corresponding nontumorous gastric mucosa in current study. The results in our study showed that SPARC expression in stromal cells surrounding gastric cancer cells nest was significantly higher than that in normal mucosa tissues. Our results also showed that the high SPARC expression was statistically significantly different with clinicopathological parameters including differentiation degree, clinical stage, Lauren classification and lymph node metastasis. The results of Siina et al. [19] were similar to that of ours.

Recently, there has been many studies about SPARC regulating tumor angiogenesis and tumor growth. One study showed that SPARC peptides have anti-angiogenic activity in vitro and in vivo. In vivo, SPARC peptides blocked angiogenesis of neuroblastoma xenografts and inhibited tumor growth [20]. Shanna A et al. [21] utilizing subcutaneous tumor models demonstrated that pancreatic tumors grown orthotopically in Sparc-null $\left(\right.$ Sparc $\left.^{-/-}\right)$mice were more metastatic than tumors grown in wild-type $\left(\mathrm{Sparc}^{+/+}\right)$littermates. It also suggested that increased tumor burden in the absence of host SPARC is a consequence of a disrupted

Table 4 Correlation analysis of SPARC and VEGF expression with MVD

\begin{tabular}{lllll}
\hline Protein expression & $\operatorname{MVD}(\bar{x} \pm s)$ & mean rank & $Z$ & $P$ \\
\hline SPARC & & & & \\
high & $40.35 \pm 8.35$ & 32.04 & -3.26 & 0.001 \\
Low & $50.15 \pm 14.33$ & 48.96 & & \\
VEGF & & & & \\
positive & $49.13 \pm 12.95$ & 48.75 & -4.45 & $<0.001$ \\
negative & $37.63 \pm 7.73$ & 24.31 & & \\
\hline
\end{tabular}

Table 5 Correlation analysis of SPARC and Ki-67 expression

\begin{tabular}{lllll}
\hline Protein expression & Ki-67( $\bar{x} \pm s)$ & mean rank & $Z$ & $P$ \\
\hline SPARC & & & & \\
high & $24.62 \pm 8.52$ & 35.36 & -1.978 & 0.048 \\
Low & $28.41 \pm 8.29$ & 45.64 & & \\
\hline
\end{tabular}

vascular basement membrane, enhanced vascular function and an immune-tolerant, pro-metastatic microenvironment. In our study, we also found that SPARC has the roles of antiangiogenesis and antiproliferation. In gastric cancer with low SPARC expression, the mean value of MVD and Ki-67-LI was significantly higher than that of cancer with high SPARC expression $(P<0.05$,respectively). Schultz $C$ et al. [22] also revealed that SPARC may promote glioma invasion but delay tumor growth in vitro and in vivo.

VEGF is an Mr 34000-42000 KD, disulfide-linked glycoprotein synthesized by several human and animal cell types, both normal and neoplastic [23]. VEGF target cell is the endothelial cell. On the other hand, VEGF stimulates the endothelial cells of microvessels to proliferate, migrate and alters their pattern of gene expression [24].The high level of VEGF expression in some malignant tumors predicts high metastasis risk and poor prognosis, such as ovarian cancer and non-small cell lung cancer[25, 26]. In current study, we found that VEGF expression highly correlated to angiogenesis, malignancy and metastasis of gastric cancer. The stronger the expression of VEGF, the higher the MVD, the lower differentiation degree, the higher clinical stage and lymph node metastasis. These results indicate that VEGF and the angiogenesis promoted by VEGF play important roles in cancer growth, infiltration and metastasis in gastric cancer.

Although the mechanism for its anti-angiogenic activity is not well understood, SPARC is capable of interfering with the binding of angiogenic stimulators such as VEGF to their receptors in endothelial cells, resulting in inhibited proliferation [6]. SPARC has also been shown to down-regulate VEGF in glioma cells [27]. Similarly, Chlenski et al. [28] demonstrated that purified SPARC potently inhibited neuroblastoma growth and angiogenesis in vivo. This is similar to our results. In our study, high levels of SPARC in stromal cells was significantly negative related with VEGF expression, the mean value of MVD and Ki-67-LI. In addition, our results revealed that the positive VEGF expression was statistically significantly different with differentiation degree, clinical stage, lymph node metastasis and Lauren classification. VEGF expression was up-regulated in gastric cancer along with the decreased expression of SPARC. All of these results suggest that SPARC may inhibit VEGF expression during the process of new blood vessel growth by which indirectly control the development, growth, invasion and metastasis of tumor cells in gastric cancer. 


\section{Conclusions}

In summary, high SPARC expression in stromal cells surrounding the tumor cell nests was related to differentiation degree, clinical stage, Lauren classification and lymph node metastasis, and may inhibit the progression of gastric cancer by anti-angiogenesis and anti-proliferation. The role of antiangiogenesis of SPARC may be involved in regulation of production of angiogenesis factor VEGF. It is believed that inhibition of SPARC expression is associated with the tumor progress and invasion process of gastric cancer. Finally, the regulatory mechanism points to the possibility that SPARCtargeted gene and protein therapy can be used as a meaningful molecular target therapy of gastric cancer.

Acknowledgment This study was supported by Grant 1155G33 from Heilongjiang province office of Education, Youth Scholar Backbone Supporting Plan Project of Heilongjiang General Colleges and Universities.

Open Access This article is distributed under the terms of the Creative Commons Attribution Noncommercial License which permits any noncommercial use, distribution, and reproduction in any medium, provided the original author(s) and source are credited.

\section{References}

1. Zhan W, Han F (2008) Surgical therapy of gastric cancer in china. J Pract Oncol 23:91-93

2. Klagsbrun MD, Amore PA (1991) Regulators of angiogenesis. Annu Rev Physiol 53:217-239

3. Dvorak HF, Brown LF, Detmer MD et al (1995) Vascualr permeability factor/vascular endothelial growth factor, microvascular hyperpermeability and angiogenesis. Am J Pathol 146:1029-1039

4. Raines EW, Lane TF, Iruela-Arispe ML et al (1992) The extracellular glycoprotein SPARC interacts with platelet-derived growth factor(PDGF)-AB and-BB and inbibits the binding of PDGF to its receptors. Proc Natl Acad Sci USA 89:1281-1285

5. Ledda F, Bravo AI, Adris S et al (1997) The expression of the secreted protein acidic and rich in cysteine (SPARC) is associated with the neoplastic progression of human melanoma. J Invest Dermatol 108:210-214

6. Hasselaar P, Sage EH (1992) SPARC antagonizes the effect of bFGF on the migration of bovine aortic endothelial cells. J Cell Biochem 49:272-283

7. Zhao Z-S, Wang Yuan-Yu (2010) SPARC Is Associated with Gastric Cancer Progression and Poor Survival of Patients. Clin Cancer Res 16(1):260-268

8. Volm M, Koomagi R, Mattern J et al (1997) Prognostic value of vascular endothelial growth factor and its receptor Flt-1 in squamous cell lung cancer. Int J Cancer 74(1):64-68

9. Weidner N, Folkman J, Pozza F et al (1992) Tumor angiogenesis:a new significant independent prognostic indicator in early-stage breast carcinoma. J Natl Cancer Inst 84(9):1875-1887

10. Ikeguchi M, Cai J, Yamane $N$ et al (1999) Clinical significance of spontaneous apoptosis in advanced gastric adenocarcinoma [J]. Cancer 85(11):2329-2335
11. Ellen JE, Sage H (1996) Regulation of angiogenesis by SPARC and angiostatin: implications for tumor cell biology. Seminars in cancer biology 7:139-146

12. Yan Q, Sage EH (1999) SPARC, a matricellular glycoprotein with important biological functions. J Histochem Cytochem 47 (12):1495-1505

13. Bradshaw AD, Sage EH (2001) SPARC, a matricellular protein that functions in cellular differentiation and tissue response to injury. J Clin Invest 107(9):1049-1054

14. Bornstein P, Sage EH (2002) Matricellular proteins: extracellular modulators of cell function. Curr Opin Cell Biol 14:608616

15. Watkins G, Douglas-Jones A, Bryce R et al (2005) Increased levels of SPARC (osteonectin) in human breast cancer tissues and its association with clinical outcomes. Prostaglandins Leukot Essent Fatty Acids 72:267-272

16. Yamanaka M, Kanda K, Li NC et al (2001) Analysis of the gene expression of SPARC and its prognostic value for bladder cancer. $\mathrm{J}$ Urol 166:2495-2499

17. Koukourakis MI, Giatromanolaki A, Brekken RA et al (2003) Enhanced Expression of SPARC/Osteonectin in the Tumor associated Stroma of Non Small Cell Lung Cancer Is Correlated with Markers of Hypoxia/Acidity and with Poor Prognosis of Patients. Cancer Res 63:5376-5380

18. Said N, Frierson JR, Chernauskas D et al (2009) The role of SPARC in the TRAMP model of prostate carcinogenesis and progression. Oncogene 28(39):3487-3498

19. Siina J, Arto K, Toru M et al (2010) Gene Expression Analysis Identifies Over-expression of CXCL1, SPARC, SPP1, and SULF1 in Gastric Cancer. Gene Chromosomes \&Cancer 49(1):28-39

20. Alexandre C, LisaJG RP et al (2010) Anti-angiogenic SPARC peptides inhibit progression of neuroblastoma tumors. Molecular Cancer 9:138

21. Shanna AA, Lee BR, Andrew FM et al (2010) Lack of host SPARC enhances vascular function and tumor spread in an orthotopic murine model of pancreatic carcinoma. Disease Models \& Mechanisms 3(1):57-72

22. Schultz C, Lemke N, Ge S et al (2002) Secreted protein acidic and rich in cysteine promotes glioma invasion and delays tumor growth in vivo [J]. Cancer Res 62(21):6270-6277

23. Berse B, Brown LF, Van D (1992) Vascular permeability factor (vascular endothelial growth factor) gene is expressed differently in normal tissues, macrophages, and tumors. Mol Biol Cell 3:210 211

24. Detmar M, Velasco P, Richard L et al (2000) Expression of vascular endothelial growth factor induces an invasive phenotype in human squamous cell carcinomas. Am J Pathol 156:159167

25. Raspollini MR, Amunni G, Villanucci A et al (2004) Prognostic significance of microvessel density and vascular endothelial growth factor expression in advanced ovarian serous carcinoma [J]. Int J Gynecol Cancer 14(5):815-823

26. Bremnes RM, Camps C, Sirera R et al (2006) Angiogenesis in non-small cell lung cancer: the prognostic impact of neoangiogenesis and the cytokines VEGF and bFGF in tumours and blood. Lung Cancer 51(2):143-158

27. Yunker CK, Golembieski W, Lemke N et al (2008) SPARCinduced increase in glioma matrix and decrease in vascularity are associated with reduced VEGF expression and secretion. Int $\mathrm{J}$ Cancer 122:2735-2743

28. Chlenski A, Liu S, Crawford SE et al (2002) SPARC is a key Schwannian derived inhibitor controlling neuroblastoma tumor angiogenesis. Cancer Res 62:7357-7363 